\title{
e-Migrinter
}

17 | 2018

Enseigner les migrations internationales

\section{La création du DEA « Migrations, Espaces et Sociétés » : extraits d'un entretien réalisé auprès de Gildas Simon}

Gildas Simon, Véronique Lassailly-Jacob et Gilles Dubus

\section{(2) OpenEdition}

Journals

Édition électronique

URL : https://journals.openedition.org/e-migrinter/1037

DOI : $10.4000 /$ e-migrinter.1037

ISSN : 1961-9685

Éditeur

UMR 7301 - Migrinter

Référence électronique

Gildas Simon, Véronique Lassailly-Jacob et Gilles Dubus, «La création du DEA « Migrations, Espaces et Sociétés » : extraits d'un entretien réalisé auprès de Gildas Simon », e-Migrinter [En ligne], 17 | 2018, mis en ligne le , consulté le 20 mai 2021. URL : http://journals.openedition.org/e-migrinter/1037 ; DOI : https://doi.org/10.4000/e-migrinter.1037

Ce document a été généré automatiquement le 20 mai 2021.

Tous droits réservés 


\title{
La création du DEA « Migrations, Espaces et Sociétés » : extraits d'un entretien réalisé auprès de Gildas Simon
}

\author{
Gildas Simon, Véronique Lassailly-Jacob et Gilles Dubus
}

En 1991, le DEA « Migrations, Espaces et Sociétés » est créé par Gildas Simon, professeur de géographie à l'Université de Poitiers, qui avait fondé quelques années auparavant, en 1985, le laboratoire Migrinter (UA 1145 -CNRS/Université de Poitiers) ${ }^{1}$. Gildas Simon a accepté avec bienveillance de raconter ses souvenirs des premières années de ce DEA. Il s'est entretenu avec nous, ce mardi 7 novembre 2017 à la MSHS de l'Université de Poitiers à propos des contextes de création, des structures et du fonctionnement de ce premier DEA dédié en France à l'étude des migrations internationales.

\section{À l'origine de la création du DEA « Migrations, espaces et sociétés "}

\section{Des raisons personnelles}

2 «Sur le fond, j'ai créé cette formation parce que j'ai toujours été personnellement préoccupé ou souhaitant en tous cas faciliter la transmission. J'ai adoré mon métier d'enseignant. C'était une forme importante de transmission mais là, en plus, l'intérêt était la transmission de la recherche, la transmission de l'intérêt pour cette question des migrations.

D'autre part, il y a plusieurs raisons personnelles. Tout d'abord, avec le recul, je peux dire que sur ce plan, ce qui m'a animé, c'est le fait que moi-même je m'étais intéressé à cette question quand j'ai réalisé ma thèse de Doctorat d'État ${ }^{2}$. Il faut rappeler que c'était une thèse longue qui a duré presque 10 ans. C'était la moyenne à l'époque. D'autres ont mis 20 ans. Pendant toute cette période je me trouvais en Tunisie mais je n'avais eu 
aucune formation méthodologique. Le sujet sur la relation migratoire entre pays d'accueil et pays d'origine était neuf. Mon patron de thèse ${ }^{3}$ était un géographe qui connaissait parfaitement l'Afrique du Nord, c'était une qualité, un appui. Mais sur les questions de migration, il n'avait pas de compétences particulières. Cet isolement m'avait fait comprendre, ensuite, l'importance d'un cadre pédagogique pluridisciplinaire pour les jeunes chercheurs. Je voulais tirer un peu le bilan de cette expérience qui m'avait été personnellement difficile sur le plan méthodologique et apporter aux jeunes chercheurs un accompagnement.

4 Ensuite, j'avais une expérience de ce qu'était un DEA, puisque j'avais participé pendant plusieurs années avec les géographes de Tours à un DEA qui a été commun aux deux universités sur le monde arabe ${ }^{4}$. J'intervenais sur les questions de migrations ou plutôt sur emploi et migration. En tant qu'intervenant j'ai appris des autres intervenants. Cette expérience collective m'a certainement été utile pour lancer quelque chose après. Je ne suis pas parti comme ça le nez au vent. J'avais déjà une certaine expérience. »

\section{Des raisons institutionnelles}

5 « Le cadre institutionnel à ce moment-là a joué. Je remue ici des souvenirs pénibles. Il y a eu ensuite une rivalité entre les deux universités de Tours et de Poitiers pour savoir qui garderait le DEA sur le monde arabe. L'équipe CNRS était à Tours et donc c'est Tours qui a gardé le DEA. Cela a été l'objet d'une forte tension très pénible pour des gens ici qui m'étaient chers. J'ai gardé un souvenir très négatif de cette expérience de rivalité entre deux établissements. A cette époque, je me suis dit qu'il fallait faire un DEA à Poitiers. Le CNRS avait reconnu l'existence de Migrinter. On avait créé aussi la REMI. Donc, cela paraissait logique de créer aussi un lieu de transmission et d'accompagnement des jeunes chercheurs. Dans la dynamique du développement de ce pôle à Poitiers, il m'a toujours semblé très important d'avoir une structure qui soit fondée sur des pieds différents mais complémentaires: L'équipe CNRS d'un côté, la Revue européenne de l'autre, et enfin le DEA, un tripode en quelque sorte. Avec trois pattes, on marche encore mieux !

6 Sur le plan institutionnel, dans ce milieu, tout est lié. D'un côté, il y a le côté institutionnel mais il y a aussi la manière dont cela fonctionne et aussi ce qui est très important c'est la qualité des relations entre les individus. Personnellement, j'ai toujours pensé qu'on ne pouvait pas faire une bonne équipe pédagogique ou scientifique si les relations humaines n'étaient pas suffisamment étroites et surtout basées sur la confiance.

7 J'ai aussi une anecdote sur le contexte institutionnel c'est-à-dire sur la perception des institutionnels vis-à-vis de ces nouvelles problématiques à l'époque. Les phénomènes étaient anciens mais les problématiques étaient récentes... Une année, en 1988, au moment du premier renouvellement de Migrinter, le laboratoire était pourtant très bien placé dans le classement interne mais le CNRS envisageait de nous dégager, de ne pas nous renouveler car il y avait des équipes bien plus anciennes et avec beaucoup de personnel qui avaient la priorité même si elles étaient beaucoup moins bien classées. Je suis allé voir le Directeur des Sciences Humaines du CNRS qui était un philosophe de formation. Quand il m'a reçu, il m'a dit: «Les migrations, ce n'est pas un objet scientifique ». Ce que j'ai contesté, évidemment. L'histoire nous a donné raison... l'histoire migratoire, l'histoire politique en France, en Europe et dans le monde. C'est 
dire à quel point cette question n'était pas du tout dans la perception ordinaire des gens qui géraient la recherche à ce moment-là. Mais c'est du temps passé. »

\section{Des raisons statutaires}

8 «Enfin, j'étais professeur de géographie et je dirigeais à cette époque le Département de Géographie. J'ai dirigé ce Département pendant 10 ans. Quand tu diriges un Département, tu as plus de marges que quand tu es simple collègue... Cela m'a facilité les choses. À ce propos, je veux souligner un aspect qui est différent aujourd'hui de ce qu'il a été. À cette époque-là, l'université était encore un monde un peu convenu avec des règles explicites et implicites, des règles qu'il fallait connaître et respecter. Mais aussi, il y avait des possibilités d'action, on n'était pas comme aujourd'hui sous une tutelle très présente. Ceux qui prenaient des initiatives pouvaient lancer des actions, à partir du moment où ils trouvaient les bases financières et qu'il y avait également un souhait de participation des enseignants-chercheurs.

9 J'ai toujours considéré que Poitiers était pour cela une bonne université. Il fallait nouer quelques contacts avec les gens qui avaient l'autorité, leur expliquer ce qu'on voulait faire et si on était motivé cela pouvait marcher. À l'époque, sur le plan institutionnel, "pour qui en avait la volonté, il y avait un chemin" en paraphrasant ici une phrase du Général de Gaulle.»

\section{Des raisons académiques : création d'une UV libre sur les migrations internationales}

10 «Ce DEA, fondé en novembre 1991, s'intitulait «Migrations, Espaces et Sociétés ». Il développait les axes de Migrinter. Je voulais garder une cohérence entre l'ensemble des composantes: les recherches du laboratoire Migrinter et les thèmes de la Revue Européenne. De plus, un certain nombre de mémoires de maîtrise avaient été faits sur le sujet. Il y avait sur place des étudiants qui désiraient persévérer dans cette voie. Cela s'est imposé comme une chose évidente, il fallait le faire... D'autant plus que dans mes enseignements de Licence j'avais proposé à mes collègues la création en 1985 d'une UV libre sur les migrations pour les étudiants de l'année de Licence. Cette UV libre a été pour l'enseignement des migrations quelque chose de capital au moins sur le plan local bien entendu. C'était une Unité de Valeur qui était ouverte aux étudiants de géographie mais aussi à ceux d'autres disciplines, les sociologues, les psychologues, les juristes... J'ai dirigé cette UV libre pendant 10-15 ans. Cette création aurait pu paraître saugrenue parce que Poitiers n'était pas une grande région d'immigration même si les travaux de construction du campus universitaire en 1968 avaient été faits par des travailleurs immigrés! Il n'y avait donc pas de logique locale ou régionale sur le sujet des migrations mais par contre l'importance et l'intérêt au niveau national étaient évidents, déjà à cette époque.

11 Pour revenir à la création de l'UV libre, j'avais essayé dans cette UV de mêler à la fois une analyse de type scientifique et en même temps de montrer la réalité des choses notamment avec l'utilisation de vidéos, de documentaires que j'avais enregistrés à la télévision et que je repassais en cours. Par exemple, un film enregistré «Pain et Chocolat » de Francesco Brusati reste pour moi un chef-d'œuvre sur le sujet. 
12 Je laissais aussi aux étudiants la possibilité de faire un dossier. C'était un petit mémoire de 40-50 pages, assez construit, assez documenté, d'un certain niveau. Dans la note de l'examen, il y avait un devoir sur table mais aussi ce mémoire pour lequel je laissais beaucoup de liberté aux étudiants. Parfois le sujet s'imposait à l'étudiant. Il y avait, par exemple, des Portugais dans la région ou des gens d'origine algérienne. Je pense que cette démarche qui oblige l'étudiant à se dire «il faut que je fasse un mémoire, il faut que je trouve un sujet » était formatrice. Cette démarche a été assez pertinente car un certain nombre - pas tous - des étudiants en sciences humaines à l'université de Poitiers ont connu à cette époque là cette UV libre. Cela a été une première sensibilisation au niveau de la Licence. Des étudiants de l'époque comme Nelly Robin, Naïk Miret, William Berthomière, Kamel Doraï ont fait cette UE libre et sont devenus par la suite membres de Migrinter.

Cette liberté dans le choix du sujet créait un lien personnel entre l'étudiant, l'enseignant et le sujet. C'était, en tant qu'enseignant, un moment de plaisir, une relation personnelle qui s'établissait au niveau de la Licence, qui n'était pas encore celle de la maîtrise ou du DEA. J'ai appris beaucoup de choses par eux. J'orientais des choix mais en même temps je laissais de la liberté. Je donnerai l'exemple de Béatrice Verquin. Je lui avais suggérée de faire quelque chose entre Foi catholique et migration. On était loin de la géographie classique! Mais c'était l'avantage d'une petite université. On avait une marge de liberté, de propositions de recherches plus ouvertes que dans des grands établissements universitaires où laisser faire un sujet comme celui-ci aurait pu provoquer un débat sur la laïcité par exemple.

De fait, dans cette UV libre, on a pris des libertés assez grandes avec les frontières ordinaires de la géographie sans renier pour autant son identité. Mais le plus important c'est que cela permettait déjà l'implication personnelle de l'étudiant. Un rapport particulier s'établissait soit avec un objet soit avec un terrain soit avec une problématique. Et ce rapport s'établissait déjà, au niveau de la licence, ce qui était précoce. Plus tard, cette démarche n'a commencé qu'au niveau de la Maîtrise puis du Master. »

Gilles Dubus souligne, à ce propos, que ces dossiers de licence ont été précieusement conservés et qu'il y en a presque 300. Il pense qu'un étudiant de Master pourrait un jour analyser les contenus de ces dossiers pour en faire le thème de son mémoire.

\section{Des raisons sociétales et politiques}

Gilles Dubus intervient pour rappeler que la période pendant laquelle Béatrice Verquin a fait ce travail devait coïncider avec les premières années d'existence des revues des Scalabrini migration centers ${ }^{5}$. Gildas Simon répond:

«Je ne pense pas qu'elles existaient à cette époque mais il y avait déjà une littérature . $^{\circ}$ Je ne connais pas l'histoire de tous les disciples de Jean-Baptiste Scalabrini mais j'en ai rencontré un qui a fait partie du premier comité de rédaction de la REMI. Il avait été cofondateur et premier directeur du CIEMI, le Centre d'Information et d'Études sur les Migrations Internationales à Paris. C'était le Père Antonio Perotti. Je lui avais demandé de faire partie du comité de rédaction de la REMI parce que je pensais que c'était important que des gens dont la mission était de s'occuper des migrants fassent partie de ce comité. Les Scalabriniens existaient depuis plus d'un siècle. Ils avaient été les pionniers dans ce domaine de la recherche, pour la foi chrétienne d'accord, mais il était 
important qu'une voix du domaine spirituel puisse s'exprimer. Antonio Perotti a fait, à ma demande, un article sur l'Église et les migrations qui est paru en $1986^{7}$.

D'autre part, en 1986, c'était la première fois que Jean-Marie Le Pen à la tête du Front National se présentait à des élections législatives ${ }^{8}$. Il avait fait un score qui avait beaucoup interrogé la classe politique et la société. De mon côté, conscient du rapport qui était en train de s'établir entre immigration et problématique sociale, j'avais pensé qu'il fallait attirer l'attention des pouvoirs publics sur la faiblesse des moyens financiers et humains pour l'étude de cette question primordiale. J'avais donc écris un article qui a été publié à l'époque dans le Monde. Il montrait le lien qui existait déjà dans l'esprit des gens qui travaillaient sur la question migratoire sur le rapport entre l'émergence du Front national et cette question migratoire et le très peu d'intérêt dont cette question migratoire faisait l'objet au niveau des pouvoirs politiques et ceci pourtant se passait sous Mitterrand.»

\section{Première équipe enseignante du DEA et premières pratiques pédagogiques}

\section{Une équipe enseignante en mouvement et pluri-disciplinaire.}

17 "C'était une équipe en mouvement. Dès le départ, les enseignants qui participaient au DEA étaient français et étrangers, je pense notamment à nos amis d'Afrique du Nord comme Mohamed Charef du Maroc ou Hassan Boubakri de Tunisie. L'équipe pédagogique était composée à la fois d'enseignants des universités de Poitiers et du Maghreb, de gens qui participaient au comité de rédaction de la REMI comme André Lebon qui travaillait à la DPM, la Direction de la Population et des Migrations au ministère du Travail ou Jacqueline Costa-Lascoux, dont on connaît les compétences en sociologie et droit des migrations. André Lebon et Jacqueline Costa-Lascoux sont venus très régulièrement.

Il y avait aussi des intervenants qui travaillaient dans l'aménagement notamment en architecture. Par exemple, Daniel Pinson, un architecte professeur à Nantes venait enseigner ici, et moi-même j'allais faire quelques interventions à Nantes. Il y avait déjà cette idée de pluridisciplinarité, d'ouverture. De l'intérieur, je me rappelle qu'au départ, le père de Kamel Doraï, sociologue à Poitiers avait participé aux débuts de Migrinter. Mais avait-il été membre de l'équipe pédagogique ? Je ne m'en souviens pas.

Il y avait surtout des gens de l'extérieur, des membres associés que j'avais sollicités. Par exemple, un de mes collègues, Jacques Guillaume, professeur sur les questions maritimes à Nantes avait été longtemps maître de conférence ici, à Poitiers et je l'avais sollicité sur les plateformes pétrolières en mer de Norvège. André Bourgey est intervenu. Il avait été directeur du CERMOC Centre d'étude et de recherche sur le Moyen Orient contemporain à Beyrouth puis avait enseigné à l'INALCO avant d'en devenir le président. Il avait participé aux premiers Cahiers de l'Orient sur les étrangers dans le Golfe. Jean-Pierre Carrière, un économiste qui travaillait à l'IUT, était venu plusieurs années, Hervé Domenach, également, Daniel Pinson, l'architecte de Nantes, Michel Poinard, Pierre Signoles au début peut-être mais il a accepté un poste de professeur à Tours par la suite. Hassan Boubakri qui est venu assez souvent, Raymond 
Pébayle, Michel Picouet, un démographe de l'ORSTOM qui travaillait en Tunisie, Dominique Royoux, aujourd'hui professeur de géographie à Poitiers, sont intervenus.

Ensuite, des chercheurs du CNRS comme Emmanuel Ma Mung et Stéphane de Tapia sont arrivés à Migrinter vers 1986 et ont intégré très vite l'équipe pédagogique. »

\section{Des pratiques pédagogiques axées sur la méthodologie et sur la transdisciplinarité}

21 «Les pratiques pédagogiques étaient très axées sur la méthodologie, sur un regard transdisciplinaire. Les étudiants appréciaient bien que ce DEA ne soit pas fondé sur une seule discipline. »

Gilles Dubus signale en outre que les présentations des retours de terrain des chercheurs étaient très appréciées des étudiants. Cela donnait beaucoup de vie. Quand il rencontrait les étudiants au centre de documentation, ils en parlaient beaucoup. Il rappelle que cela fait plus de 20 ans qu'il participe au DEA par des enseignements sur la méthodologie des recherches bibliographiques et de l'ensemble des ressources documentaires. Gildas Simon affirme ...

« Des personnes comme Gilles Dubus, documentaliste à Migrinter, ont beaucoup aidé les étudiants. Son rôle était de les aider à faire du repérage dans la documentation, de leur signaler des pistes, d'intervenir sur les questions méthodologiques. C'était très précieux pour les étudiants. J'en profite pour dire que l'arrivée de Gilles Dubus en 1993 a inauguré une nouvelle ère pour Migrinter. On avait une approche assez traditionnelle ici. Il nous a fait prendre très aisément le tournant de l'ère de la numérisation et des techniques scientifiques en matière de documentation. La plupart des gens ont gardé un bon souvenir de leur séjour ici grâce à Gilles qui, par son statut, a toujours été un permanent C'est lui qui fait le lien entre les étudiants et l'équipe pédagogique. Il a toujours été l'un des piliers de Migrinter et du DEA. Les étudiants venaient d'euxmêmes solliciter le documentaliste. C'était une rencontre incontournable. Autrefois, dans les anciens locaux, le centre de documentation était le lieu central du Laboratoire. »

Gilles Dubus souligne à ce propos la différence entre hier et aujourd'hui. Il constate qu'aujourd'hui les étudiants n'ont plus le réflexe de solliciter le documentaliste. Ils ont le réflexe des sources numériques. Ils sont beaucoup sur internet. Depuis quelques années, ils sont moins demandeurs de conseils méthodologiques.

\section{L'importance du centre de documentation}

Gilles Dubus rappelle que le laboratoire Migrinter a déménagé à la MSHS, en 1996 et que le centre de documentation a été installé en 1998 dans la nouvelle bibliothèque. Gildas Simon raconte :

«J'ai été un des fondateurs de la MSHS avec Éric Esperet qui a été ensuite président de l'Université. Il nous fallait aménager ces nouveaux locaux. J'avais été invité à l'UCLA, l'Université de Californie à Los Angeles et à Berkeley dans ces années-là. Les collègues de Berkeley m'avaient fait visiter leur bibliothèque de recherche. J'avais été sidéré de voir l'ampleur des locaux et surtout la capacité d'action qu'ils avaient et sans doute de finances. Quand il a été question d'aménager la MSHS et d'y installer le centre de Documentation, j'ai fait valoir l'importance d'avoir une bibliothèque intégrée. Il était 
clair que pour un DEA sur les migrations comme pour une revue transdisciplinaire, il était indispensable d'avoir une bibliothèque intégrée. Il n'était pas question de cloisonner les disciplines. Mais ce n'était pas évident à l'époque. »

\section{En 1993, l'évolution du DEA vers une co-habilitation avec l'université Paris-Diderot.}

\section{Une co-habilitation enrichissante}

«On a franchi un cap ensuite en 1993. Cette année-là, j’ai demandé à Maryse Tripier, que je connaissais par mon détachement pendant ma thèse à l'équipe de recherche sur les migrations internationales basée à Paris, l'URMIS actuel si elle serait d'accord pour faire un DEA à double implantation et double repères disciplinaires : notre DEA à Poitiers fondé plus sur l'analyse spatiale que sur la géographie et un DEA à l'université Paris-Diderot fondé sur l'approche sociologique autour de l'URMIS. Ce qui était intéressant dans cette création, c'est qu'il était désormais possible, statutairement, de faire porter un DEA par deux universités. La nouvelle politique institutionnelle du Ministère de l'enseignement supérieur et de la recherche (dont François Fillon était le ministre de 1993 à 1995) était de dire que c'était désormais possible. Après la stricte législation d'Alice Saunier Seïté, on avait laissé respirer les gens. Il n'y avait donc plus de problèmes au niveau institutionnel. Maryse a accepté tout de suite cette proposition de travail en commun. Ce DEA s'intitulera «Migrations internationales et relations interethniques ». On avait créé - ce à quoi je tenais beaucoup - la double obligation pour les étudiants de suivre des cours à la fois à Poitiers et à Paris. Il y avait sans doute une semaine de cours à Paris puis une semaine de cours à Poitiers. Je ne sais plus quels étaient les aspects concrets. Mais c'était impératif pour nous. L'idée sous-jacente était d'amener les étudiants à circuler entre des lieux de recherche et de production scientifiques différents. De les obliger. C'était très directif. J'ai toujours été un peu directif. Et là, ils étaient obligés...

Cela a aussi permis aux étudiants qui fonctionnaient dans ce double système de créer des liens personnels beaucoup plus forts entre Parisiens et Poitevins. Cela représentait un coût financier pour eux puisqu'il fallait payer le train et en même temps il fallait trouver un logement des deux côtés. Donc, les uns logeaient les autres et vice-versa. Ce va-et-vient était bien apprécié par les étudiants et créait des liens. Les étudiants constituaient une équipe un peu diversifiée qui apprenait à dialoguer avec d'autres. Il y avait une très bonne ambiance dans cette équipe.

J'ai une anecdote sur cette période. Comme on avait une politique de communication assez efficace, on avait envoyé nos affiches de DEA dans presque toutes les universités françaises où elles étaient placardées dans les lieux administratifs. Je me rappelle d'une étudiante qui habitait la Corse. Elle était tombée en arrêt devant l'une de ces affiches et s'était dit que c'était cela qu'elle voulait faire. Donc, elle "triangulait" pendant toute l'année universitaire entre la Corse où elle résidait, Paris et Poitiers. C'était vraiment une circulation migratoire au sens scientifique du terme. Je crois que les gens qui ont vécu cette époque en ont gardé plutôt un bon souvenir.

L'examen était commun. Il portait d'une part sur les enseignements donnés à Paris Diderot et d'autre part sur les enseignements donnés à Poitiers. Il y avait à la fois un devoir de sociologie et un devoir en géographie. Il y avait également une appréciation 
qui était faite sur les dossiers de DEA. Le jury était commun. Soit j'allais à Paris soit les Parisiens venaient ici. En gros cela se passait comme cela.

Ce DEA commun nous a donné une capacité de rayonnement assez importante. Paris, cela va de soi, et Poitiers avait cette spécialisation déjà connue. Le "bouche à oreille » fonctionnait bien. C'est une époque où on a eu pas mal de demandes d'inscription. De même, au départ, la sélection, car il y avait une sélection sur les dossiers de candidature, se faisait en commun. Mais là encore, le lien humain était fondamental. On s'entendait très bien, on était conciliant les uns avec les autres, on se faisait confiance. »

\section{Le déclin des étudiants étrangers notamment originaires du Maghreb}

Gilles Dubus observe une constante diminution du nombre d'étudiants étrangers. Il souligne que la rupture a eu lieu lorsque Nicolas Sarkozy, ministre de l'intérieur, a durci les modalités d'entrée des étudiants étrangers. Avant ce durcissement dû à Nicolas Sarkozy il y avait dans chaque promotion, au moins deux Marocains. Par la suite, cette proportion d'étudiants d'Afrique $\mathrm{du}$ Nord s'est asséchée presque totalement. Les étudiants marocains ont presque tous disparu. Gildas Simon se souvient :

« Déjà dans le DEA Monde Arabe à Tours, on bénéficiait de cette présence des étudiants maghrébins. Quand le DEA a été créé à Poitiers, cela s'est amplifié. A cette époque, ce n'était pas difficile pour les étudiants étrangers d'obtenir un passeport puis une autorisation d'entrée. Les administrations locales étaient bienveillantes envers les étudiants. Cette disparition des étudiants maghrébins est un changement total par rapport à ce que j'ai connu et par rapport aux thèses et mémoires que j'ai dirigés. Il serait d'ailleurs intéressant de relever toutes les thèses d'étudiants maghrébins qui ont été soutenues avec moi à Poitiers. Il y avait une assez grande proportion d'étudiants Marocains. Par contre il n'y avait que très peu d'Algériens. Je pense qu'ils allaient ailleurs, à Marseille, à Aix ou à Paris. Et il y avait peu de Tunisiens. Les Marocains étaient les plus présents, les plus intéressés. Mohammed Charef, aujourd'hui professeur de géographie à l'Université d'Agadir, a fait une thèse de 3ème cycle puis une thèse d'État. C'était le premier de ceux qui sont passés par ici à avoir un poste de professeur. Il y avait très peu d'étudiants des pays subsahariens à cette époque.

31 Par contre, nous avons eu en DEA un étudiant irakien parti sous le régime de Saddam Hussein. Cet étudiant était intéressé par les conditions de travail des étrangers au Koweit. Il voulait faire son DEA sur un vrai sujet c'est-à-dire en gros sur $85 \%$ de la population vivant au Koweit. Ils faisaient tous les sales boulots. Ils étaient traités de manière infâme. Cet étudiant voulait aller faire sa recherche à Koweit City. Il lui fallait l'accord de l'ambassade de France au Koweit. Un jour, je reçois une lettre incendiaire de l'ambassadeur de France à Koweit qui refuse l'autorisation de recherche sur cette question. L'entrée lui a été refusée. Et pourtant il n'était pas un terroriste mais le sujet était totalement tabou localement. C'était la première fois de manière aussi explicite qu'une ambassade française faisait obstacle à des recherches à l'étranger. C'était un sujet qu'il ne fallait pas toucher. On touche ici du doigt les réalités du monde social international ». 


\section{Conclusion : l'enseignement des migrations internationales : une problématique devenue cruciale}

«Sur le fond, sur l'ensemble de la question de l'enseignement des migrations internationales, je pense que c'était vraiment une problématique importante. Au départ, on avait l'impression d'être un peu des pionniers. Cela ne voulait pas dire que dans telle ou telle faculté, il n'y avait pas des recherches menées sur ce sujet. Il y en avait eu à Nice, à Rouen. Ce qui était la preuve qu'il y avait déjà un intérêt sur cette question. Mais ce que j'observe maintenant, c'est que cette nécessité sociétale d'un enseignement, d'un apprentissage de cette question, on l'observe aujourd'hui à l'échelle nationale et pas seulement à l'université. Je pense que cette question est traitée désormais dans l'ensemble de l'enseignement universitaire. Et cette pédagogie se trouve aussi proposée à l'échelle de l'enseignement secondaire et peut-être même primaire. Les enjeux de société sont tels que ce qui était une évidence pour quelquesuns est devenu une évidence pour un grand nombre d'enseignants ou de responsables d'éducation. Il y a eu une évolution considérable des mentalités.

Cette question des migrations internationales dans le monde est également apparue au programme de l'agrégation de géographie. J'ai été au jury pendant 2 ou 3 ans. Cela a contribué à ce que cette question qui était considérée comme une fantaisie au début et une originalité ensuite, s'impose aujourd'hui comme une évidence, en France comme dans la plupart des pays européens. Voici une anecdote qui concerne l'agrégation et dont je me rappellerai toujours: j'avais un collègue ici qui était dans l'équipe d'un laboratoire de psychologie. Je ne sais plus très bien l'historique exact mais il avait suivi la réalisation du projet de la MSHS. Il devait être dans les petits papiers du Ministère puisqu'il avait été nommé conseiller du Ministre de la Recherche scientifique.

Un jour, dans les anciens locaux, il m'appelle et me dit :

Gildas, je te nomme président du jury de l'agrégation de géographie.

lui propose Daniel Noin qui avait été dans mon jury de thèse. Daniel Noin a été nommé président. C'est lui qui a mis la question au programme parmi d'autres. Pourquoi ? Tout est lié. Avec Daniel Noin, nous avions fait le premier article scientifique de notre génération sur les migrations maghrébines qui était paru dans les Cahiers d'Outre-Mer en 1972. Je l'avais rencontré par l'intermédiaire des études sur le monde arabe. On était toujours resté en contact. In fine, les migrations ont été mises au programme de l'agrégation. Le sujet est tombé mais je n'y suis pour rien. Les commissions qui se réunissent proposent différents sujets mais le président décide seul du sujet. Le fait que les migrations soient devenues un sujet d'agrégation est un fait important. C'était en quelque sorte une réponse au directeur des Sciences Humaines du CNRS. C'était important pour l'évolution des mentalités, pour la prise de conscience des établissements scolaires et universitaires... »

Véronique Lassailly-Jacob et Gilles Dubus remercient chaleureusement Gildas Simon pour cet entretien passionnant qui apporte un éclairage très complet sur les premières années du DEA « Migrations, espaces et sociétés ». 


\section{NOTES}

1. À cette époque, le laboratoire Migrinter s'intitulait «Migrations internationales de travail et sociétés d'origine ».

2. «L'espace des travailleurs tunisiens en France. Structure et fonctionnement d'un champ migratoire international » thèse d'État soutenue par Gildas Simon en 1979.

3. Gérard Maurer, professeur de géographie à l'Université de Poitiers.

4. En 1977 était créée à l'université de Tours l'ERA 706 (Équipe de recherche associée au CNRS) intitulée «Urbanisation, réseaux urbains et régionalisation au Maghreb » qui devint en 1982 le laboratoire Urbama, le centre d'études et de recherches sur l'urbanisation du monde arabe.

5. Giovanni Battista Scalabrini, évêque italien de la fin du XIXème siècle, considéré comme le père des migrants. Un réseau des centres d'études Giovanni Battista Scalabrini est ensuite créé. C'est en 1975 que la première revue « Sur les chemins de l'exode » parait en Italie suivie en 1989 par la revue Migrations et Société.

6. Depuis 1963, la congrégation scalabrinienne possède à Rome un Centre d'études sur les migrations, le CSER.

7. Autre publication de Perotti Antonio (dir.) : L'Église et les migrations. Un précurseur: Giovanni Battista Scalabrini, Paris, Éd. CIEMI - l'Harmattan, 1999.

8. Lors des élections législatives de 1986, le parti du Front National (FN) fondé par Jean-Marie Le Pen en 1972, entre à l'Assemblée nationale avec 35 députés et un score de 9,7 \%. C'est en 1986 que le FN émerge vraiment sur le devant de la scène politique française.

\section{INDEX}

Index géographique : France

Mots-clés : enseignement supérieur, entretien

\section{AUTEURS}

\section{GILDAS SIMON}

professeur de géographie émérite - Université de Poitiers, laboratoire Migrinter

\section{VÉRONIQUE LASSAILLY-JACOB}

professeur de géographie émérite - Université de Poitiers, laboratoire Migrinter

\section{GILLES DUBUS}

Documentaliste - CNRS, Laboratoire Migrinter

gilles.dubus@univ-poitiers.fr 